\title{
Coupled flow and anisotropy in the UltraLow Velocity Zones
}

\author{
Saswata Hier-Majumder ${ }^{\mathrm{a},}$, Tyler W Drombosky ${ }^{\mathrm{b}}$ \\ ${ }^{a}$ Department of Earth Sciences, Royal Holloway University of London, Egham, Surrey, \\ TW20 OEX, UK. \\ ${ }^{b}$ Luminal, Inc., 47 E All Saints St, Frederick, MD 21701
}

\begin{abstract}
Seismic observations reveal a patchwork of thin and dense structures, named UltraLow Velocity Zones (ULVZs) atop the Earth's core mantle boundary. The high width to height ratio of the ULVZs, their spatial correlation with the edges of Large Low Shear Velocity Provinces (LLSVPs), and their preservation as distinct structures in the convecting mantle remains an enigmatic problem. In this article, we carry out a series of numerical simulations using Fast Multipole Boundary Elements Method (FMBEM) to address these questions and study the internal deformation within the ULVZs. Our results demonstrate that coupled flow between dense, low viscosity ULVZ patches and the LLSVP accumulates the ULVZ into stable piles along LLSVP corners, while coalescence and gravitational drainage leads to thin and wide ULVZs away from the corners. Deformation of the matrix is localized within the weaker ULVZ and the LLSVP edges, while the strain in the interior of the LLSVP remain uniform and low, explaining the observed localized anisotropy near LLSVP edges.
\end{abstract}

Email address: Saswata.Hier-Majumder@rhul.ac.uk (Saswata Hier-Majumder) 
Keywords: Core-Mantle Boundary; Two-Phase Flow; ULVZ; LLSVP; Mantle Heterogeneity

\section{Introduction}

The base of the Earth's mantle is marked by a patchwork of seismically 3 anomalous structures named UltraLow Velocity Zones (ULVZs), most com4 monly observed along the edges of two larger antipodal structures, termed ${ }_{5}$ Large Low Shear Velocity Provinces (LLSVPs). The ULVZs are typically 8 6 to $10 \%$ denser than the surrounding mantle and are marked by up to $30 \%$ and $710 \%$ reductions in shear and compressional wave speeds, respectively (Rost 8 et al., 2005; Rost and Revenaugh, 2003; Williams and Garnero, 1996). Nar9 row, elongated ULVZs can span up to several hundreds of kilometers in length 10 while reaching only a few tens of kilometers of height above the CMB (Cot11 taar and Romanowicz, 2012; Rost et al., 2005; Rost and Revenaugh, 2003; 12 Thorne et al., 2013; Williams and Garnero, 1996). The larger LLSVPs, characterized by up to $3 \%$ reduction in shear wave speed, are dynamic and likely chemically distinct structures (McNamara et al., 2010). Despite strong internal circulation and deformation (Bower et al., 2011; McNamara et al., 2010), seismic anisotropy is strongly localized along the margins of the LLSVP (Cottaar and Romanowicz, 2013; Lynner and Long, 2014).

A number of issues arise in reconciling the physical characteristics and location of the ULVZs with the available constraints. The distinct physical properties and patchy occurrence of the ULVZs suggest that they are compositional, rather than thermal anomalies. The excess density and large shear wave speed reduction within the ULVZs are likely caused by $5-10$ vol\% 
neutrally buoyant melt (Fiquet et al., 2010; Hier-Majumder, 2014; Wimert and Hier-Majumder, 2012) hosted in an iron-rich matrix (Mao et al., 2006; Muir and Brodholt, 2015a,b; Nomura et al., 2011; Wicks et al., 2010). Analogue material experiments and geodynamic calculations suggest that the ULVZs are likely two orders of magnitude weaker than their surrounding (Hier-Majumder and Revenaugh, 2010; Jellinek and Manga, 2004), rendering them susceptible to mixing with the ambient mantle (Manga, 1996). The ULVZs can serve as isolated and enriched reservoirs at the base of the mantle as required by Sm-Nd isotopic measurements (Boyet and Carlson, 2005; Coltice et al., 2011). Based on the seismic observations and these constraints, the outstanding issues are: (a) the high aspect ratio of the ULVZ patches (width is 45-80 times higher than the height (Cottaar and Romanowicz, 2012; Thorne et al., 2013)) and their preferential associations with the corner of the LLSVPs, (b) the preservation of the low viscosity ULVZs as distinct structures over geologic timescales, and (c) the fact that the flow in the LLSVP does not produce internal seismic anisotropy. In this article, we demonstrate that strongly coupled flow in the ULVZ-LLSVP system stabilizes dense ULVZs at the LLSVP corners while stretching them to small thicknesses in the other regions. We also demonstrate that matrix deformation arising from this coupled flow is strongly partitioned into the weaker ULVZ interior and along the LLSVP edges.

To date, majority of the discovered ULVZ locations are associated with edges of the Pacific and the African LLSVPs. There are, however, observation of ULVZ patches within a 'hole' inside the Pacific LLSVP (Thorne et al., 2013) and isolated ULVZ patches away from the LLSVP (e.g. McNa- 
mara et al., 2010, and references therein). The occurrence of these ULVZs can be explained by lateral migration and coalescence of segments of the LLSVP, spillage of ULVZ material outside the LLSVP, or the presence of ULVZ material inside and outside the LLSVPs. In this work, we do not address these processes and focus, instead, on the more commonly observed instance of the association between the LLSVP edges and the ULVZs.

Tracking the evolution of the ULVZ shape while monitoring the internal deformation has been difficult in the existing scope of work. While analog material experiments are able to provide a wealth of information on the evolution of the ULVZ topography, a quantitative description of internal deformation is unattainable in these experiments (Jellinek and Manga, 2004). In contrast, high resolution convection models can quantify and map the strain within the ULVZ, but due to the lack of an explicit interface between the ULVZ and the ambient mantle, tracking the shape of the ULVZ and calculating the viscous drag on the ULVZ surface are not straightforward in these models (Bower et al., 2011; McNamara et al., 2010).

In this article, we present a new geodynamic model that tracks the shape and interaction between multiple ULVZ patches while monitoring their internal velocity and strain fields. The system is driven by the flow within the LLSVP. The circulation within the LLSVP and the ULVZs are modeled using a Fast Multipole Boundary Elements Model (FMBEM). A detailed description of the governing equations, boundary conditions, and the numerical techniques are presented in the online supplementary material. In this model, the velocity fields are calculated using a set of coupled boundary integral equations, accelerated by fast multipole expansion of the integral 


\section{Methods}

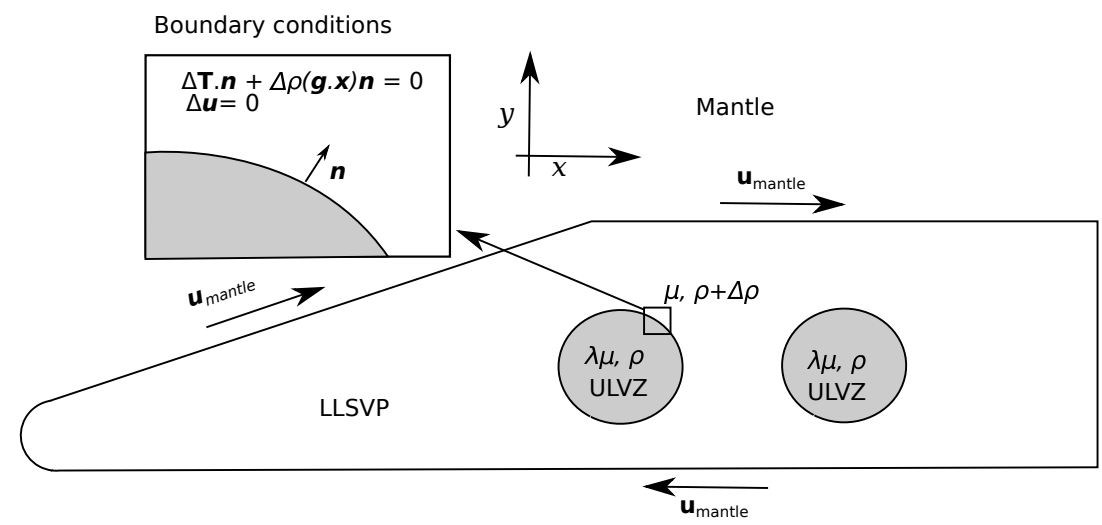

Figure 1: A schematic diagram outlining the scope of the problem. The ULVZ patches within the LLSVP are shaded in light gray. Density and viscosity within each of the entities are shown in the figure. The figure in the inset shows the no-slip and stress jump boundary conditions at the ULVZ-LLSVP interface. Velocity at the LLSVP-Mantle interface are shown by the arrows marked by $\boldsymbol{u}_{\text {mantle }}$.

kernels (Liu and Nishimura, 2006). As the shape of the ULVZ, the velocity, and pressure fields are variables in this system, quantitative evaluation of the shape and internal deformation can be performed directly.

In our model, we treat the ULVZs as dense, low viscosity patches, embedded within a larger LLSVP. In our calculation in two-dimensional Cartesian coordinates, the coupled convective flow within the LLSVP and the ULVZs are driven by density contrast between the LLSVP and the ULVZ patches and the prescribed velocity at the boundary between the LLSVP and the surrounding mantle.

The numerical model in this work builds on a previous boundary elements model developed to study the microstructure in partially molten 
rocks (Drombosky and Hier-Majumder, 2015; Hier-Majumder, 2008; HierMajumder and Abbott, 2010; Hier-Majumder and Drombosky, 2015). In this method, the governing partial differential equations and the boundary conditions are combined into a vector Boundary Integral Equation (BIE). In the next subsection, we present the governing equations.

\subsection{Governing equations}

We treat the ULVZs and LLSVP as individual bodies of incompressible fluid, with distinct densities and viscosities. Conservation of mass within each entity requires,

$$
\nabla \cdot u=0
$$

where $\boldsymbol{u}$ is the velocity. Conservation of momentum within each ULVZ and the LLSVP requires, in the presence of gravitational acceleration,

$$
\nabla \cdot \mathbf{T}+\rho \boldsymbol{g}=0
$$

where $\mathbf{T}$ is the stress tensor of the fluid with density $\rho$ and the vector $\boldsymbol{g}$ represents gravity. The schematic diagram in Figure 1 outlines the boundary conditions associated with the coupled flow within each of these bodies. The governing conservation equations must be supplemented with boundary conditions. We impose a no-slip boundary condition at the interface between the ULVZ and the LLSVP, expressed as,

$$
\Delta \boldsymbol{u}=0
$$

02 where $\Delta q$ indicates the difference between the physical quantity $q$ evaluated within the ULVZ and evaluated within the LLSVP. In addition, we impose a stress jump boundary condition across each interface, such that the difference 
in traction is balanced by buoyancy contrast between the ULVZ and the LLSVP. This condition is given by,

$$
\Delta \mathbf{T} \cdot \hat{\boldsymbol{n}}+\Delta \rho(\boldsymbol{x} \cdot \boldsymbol{g}) \hat{\boldsymbol{n}}=0,
$$

where $\hat{\boldsymbol{n}}$ is the unit normal to the interface, as depicted in Figure 1.

We solve for velocities at the boundary and within each entity by converting the governing partial differential equations into a Boundary Integral Equation (BIE). We provide the details of this conversion, the nondimensionalization scheme, and implementation of the fast multipole expansion in the supplementary material. For the sake of brevity, we next proceed to the integral equation describing the coupled flow over multiple domains.

In a collection of $P$ ULVZ patches embedded within an LLSVP (marked as domain $P+1$ ), the dimensionless BIE for the velocity $\boldsymbol{u}$ at point $\boldsymbol{x}_{0}$ on the surface of the $q$-th ULVZ patch is given by:

$$
\begin{aligned}
\boldsymbol{u}\left(\boldsymbol{x}_{0}\right)= & \frac{2}{1+\lambda_{q}}\left[-\sum_{p=1}^{P+1} \frac{\mathcal{R}}{4 \pi} \int_{\Gamma_{p}} \mathbf{J}\left(\boldsymbol{x}, \boldsymbol{x}_{0}\right) \cdot \Delta \boldsymbol{f}(\boldsymbol{x}) \mathrm{d} \Gamma_{p}\right. \\
& \left.+\sum_{p=1}^{P+1} \frac{1-\lambda_{p}}{4 \pi} \int_{\Gamma_{p}} \boldsymbol{u}(\boldsymbol{x}) \cdot \mathbf{K}\left(\boldsymbol{x}, \boldsymbol{x}_{0}\right) \cdot \hat{\boldsymbol{n}}(\boldsymbol{x}) \mathrm{d} \Gamma_{p}\right],
\end{aligned}
$$

where the point $\boldsymbol{x}_{0}$ is called the pole point while the point $\boldsymbol{x}$ is called the field point (Pozrikidis, 2001), $\lambda_{q}$ is the viscosity ratio between the $q$-th ULVZ patch and the LLSVP, $\mathcal{R}$ is the nondimensional compositional Rayleigh number, $\Delta \boldsymbol{f}(\boldsymbol{x})$ is the buoyancy force arising from density contrast between the ULVZ and the LLSVP, and the kernels $\mathbf{J}\left(\boldsymbol{x}, \boldsymbol{x}_{0}\right)$ and $\mathbf{K}\left(\boldsymbol{x}, \boldsymbol{x}_{0}\right)$ in the two integrals on the right hand side are known as the Stokeslet and the Stresslet tensors. Each of these integrals are taken over the boundary of the $P$-th domain.

The system of linear algebraic equations arising from discretization of (5) is dense and generally asymmetric. Following the methods outlined in 
Drombosky and Hier-Majumder (2015) and Hier-Majumder and Drombosky (2015), we use the Fast Multipole (FM) expansion to approximate the kernels in the integrands. After discretization and implementing FM expansion, we can recast the BIE as a system of algebraic equations given by

$$
\left[\left(\frac{1+\lambda}{2}\right) \mathbf{I}-\left(\frac{1-\lambda}{4 \pi}\right) \mathcal{K}\right] \boldsymbol{u}=-\frac{\mathcal{R}}{4 \pi} \mathcal{J} \Delta \boldsymbol{f}
$$

where $\mathcal{J}$ and $\mathcal{K}$ are the matrices generated from the kernels $\mathbf{J}\left(\boldsymbol{x}, \boldsymbol{x}_{0}\right)$ and $\mathbf{K}\left(\boldsymbol{x}, \boldsymbol{x}_{0}\right)$, respectively. The accelerated multiplication, in conjunction with iterative methods such as the GMRES (Saad, 2003), solves the linear system of equations in $(6)$ in $\mathcal{O}(N)$ time.

The nondimensional BIE consists of two dimensionless quantities, $\mathcal{R}$, the compositional Rayleigh number and $\lambda$, the viscosity ratio. We define $\mathcal{R}$ as,

$$
\mathcal{R}=\frac{\Delta \rho g x_{c}^{2}}{u_{c} \mu},
$$

where $\mu$ is the viscosity of the LLSVP, $x_{c}$ is the characteristic length, and $u_{c}$ is the characteristic velocity, respectively. The compositional Rayleigh number is the inverse of the intrusion number sometimes used in nondimensional models of spreading viscous gravity currents (Hier-Majumder and Revenaugh, 2010; Olson, 1990). It signifies the ratio between buoyancy and viscous drag. If the magnitude of $\mathcal{R}$ is high, buoyancy dominates over viscous resistance to flow and the ULVZ patches drain rapidly. Notice also that the sign of $\mathcal{R}$ is controlled by the difference between densities of the ULVZ and the LLSVP. We present further discussion about the relation between these two competing forces in Section 2.2. We discuss the value of $\mathcal{R}$ in our simulations in Section 2.3. 
Once the boundary velocities are calculated by solving equation (6), and the velocities within the ULVZ patches and the LLSVP are calculated by the methods outlined in the supplementary section, we update their shapes. We describe the shape of the $P$-th ULVZ patch by the function $F^{p}(\boldsymbol{x}, t)=0$ at time $t$. To ensure the volume of each grain remains constant during deformation of the grain, we use the kinematic equation

$$
\frac{\partial F^{P}(\boldsymbol{x}, t)}{\partial t}+\boldsymbol{u}^{P}(\boldsymbol{x}, t) \cdot \nabla F^{P}(\boldsymbol{x}, t)=0 .
$$

Using the updated shape functions, we calculate the normal vectors at each node of the new ULVZ-LLSVP boundary to be used in the BIE (5) for calculating the boundary velocities at the next time step. This process is iterated in time to model the evolution of the ULVZ shape.

\subsection{Linear analysis}

The rate of gravitational drainage of the dense ULVZs is modulated by the viscosity ratio between the ULVZ and the mantle. To understand the nature of the relationship between the two key nondimensional parameters, $\mathcal{R}$ and $\lambda$, we carried out a linear stability analysis for the ULVZ shape subjected to a straining and drainage flow.

The linear solution consists of a base state and a perturbed state. The base state is characterized by zero motion within a neutrally buoyant, hemispherical ULVZ. The perturbation is caused by both density contrast and flow induced by circulation within the LLSVP. We want to characterize, to the first order, a relation between the two nondimensional parameters, $\lambda$ and $\mathcal{R}$, to identify the boundary between two regimes, gravitational drainage and entrainment. The set up for the linear problem is outlined in Figure 2. Note 
that this analysis is applicable to the stability of the ULVZ far away from the LLSVP corners.

With this goal, we can write the entire velocity fields within the ULVZ and the LLSVP as $\boldsymbol{u}^{j}=\epsilon\left(\boldsymbol{u}_{e}^{j}+\boldsymbol{u}_{d}^{j}\right)$, where $\epsilon \ll 1$ is a constant, the superscript $j$ refer to either the ULVZ $(U)$ or the LLSVP $(L)$, and the subscripts $e$ and $d$ refer to two linear flows arising from entrainment and drainage, respectively.

To build analytical solutions for each perturbing flow, we use the method of solid spherical harmonics. We use the well-known Hadamard-Rybczynski solution for gravitational settling of a viscous drop (see, for example, Leal, 1992, Ch. 5). For the entraining flow, we build a set of solutions forced by a straining flow in the LLSVP, $\boldsymbol{u}_{e}^{L} \propto \dot{\mathbf{E}} \cdot \boldsymbol{r}$, where $\dot{\mathbf{E}}$ is a constant strain rate and $\boldsymbol{r}$ is the distance from the ULVZ center. We present a detailed description of the method for solution building in Appendix A.

For a small perturbation $f$, the shape of ULVZ is given by $F=r-h(1+$ $\epsilon f)$, where $f$ is the perturbed shape function. The $\mathcal{O}(\epsilon)$ kinematic condition (8), then becomes,

$$
\frac{\partial f(\boldsymbol{x}, t)}{\partial t}=\frac{1}{h} \boldsymbol{u}^{U}(\boldsymbol{x}) \cdot \hat{\boldsymbol{n}},
$$

where $\hat{\boldsymbol{n}}$ is the unit normal to the hemispherical ULVZ. We present a schematic diagram for the linear analysis in Figure 2(a) and solutions to equation (9) in Section 3.1.

\subsection{Simulations}

The geometry of the simulation is divided into two sets of interfaces: the LLSVP-mantle interface and LLSVP-ULVZ interfaces. For each simulation, we fix the LLSVP-mantle boundary as a trapezoid with rounded corners. 
Roundness around the corner prevents singularities in the simulation arising from high curvature of the boundary elements. The width, height, interior angle, and radius of curvature for the corners are specified to create the LLSVP geometry similar to structures observed in whole-mantle models. The LLSVP is $1,000 \mathrm{~km}$ across and $100 \mathrm{~km}$ high. The lower acute corner of the trapezoid has an angle of $\tan ^{-1}(1 / 3)$. Both the corners are rounded with a 10 $\mathrm{km}$ radius of curvature. The density of the LLSVP is $\rho_{L}=3500 \mathrm{~kg} / \mathrm{m}^{3}$, while the density of the ULVZ, $\rho_{U}$, is $3850 \mathrm{~kg} / \mathrm{m}^{3}$. The viscosity of the LLSVP is $\mu_{L}=5 \times 10^{20} \mathrm{~Pa}$ s, and the viscosity of the ULVZ is two orders of magnitude less at $\mu_{U}=5 \times 10^{18} \mathrm{~Pa}$ s. Acceleration due to gravity is $g=9.8 \mathrm{~m} / \mathrm{s}^{2}$. We impose a tangential velocity of $1 \mathrm{~cm} / \mathrm{yr}$ at the LLSVP-mantle interface.

We simulate three stages of ULVZ evolution within the LLSVP. Each simulation begins with ULVZs configured in a unique initial geometry. The first stage simulates the evolution of ULVZ material distributed in the center of the LLSVP. The second simulation begins with the ULVZ material distributed along the CMB. The third and final simulation demonstrates the steady state behavior of the ULVZ material in the corner of the LLSVP.

We set the characteristic length $x_{c}=25 \mathrm{~km}$ and the characteristic velocity $u_{c}=1 \mathrm{~cm} / \mathrm{yr}$, resulting in a time scale of 2.5 Ma. Taken together with the physical parameters for the density, gravitational acceleration, and viscosity of the LLSVP, the dimensionless compositional Rayleigh number at the LLSVP-ULVZ boundary is $\mathcal{R}=-13.5$, where the negative sign indicates that the ULVZ is more dense than the surrounding LLSVP.

These numerical simulations using the FM accelerated BEM allows us to specifically address the three questions raised in the introduction. In the 
following section, we present our results in light of these questions.

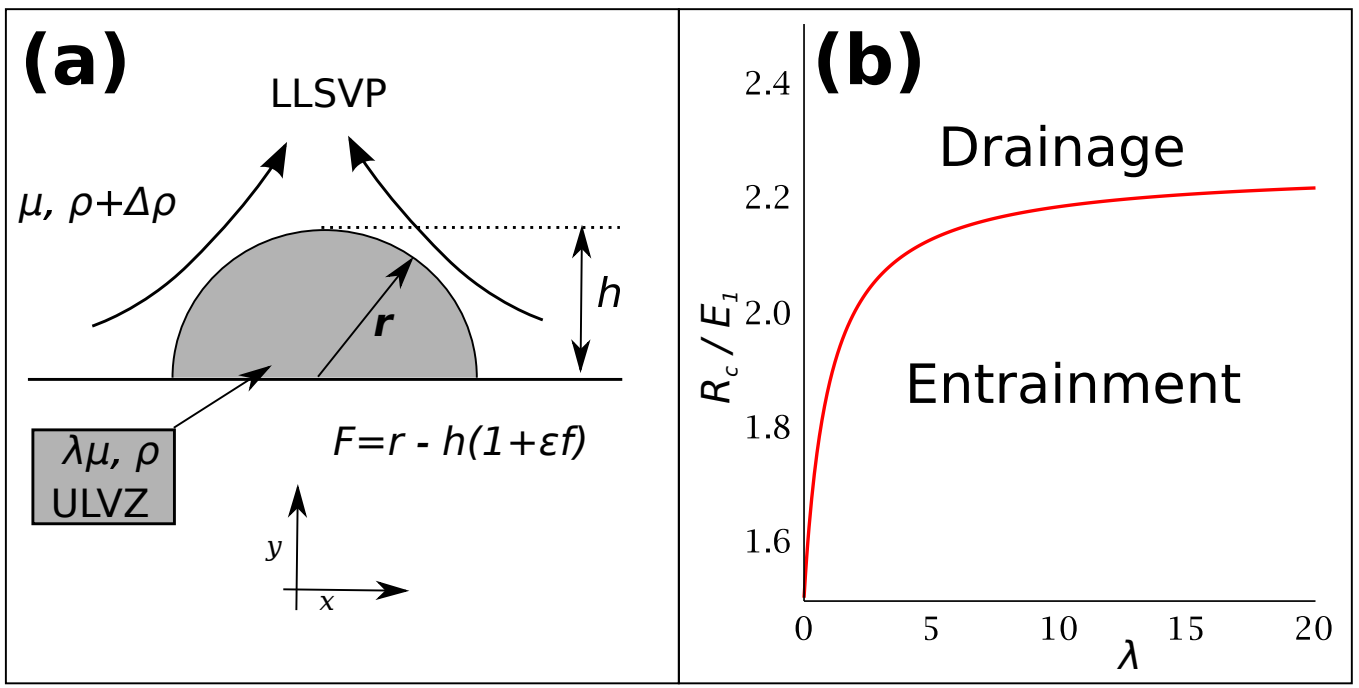

Figure 2: Linear analysis of drainage within the ULVZ. (a) A schematic diagram outlining the problem set-up for the linear analysis. (b) Plots of $R_{c} / E_{1}$ from equation (11) as a function of $\lambda$. In the plot we assume $h=1$.

As discussed in section 2.2, we build solutions for the entraining flow in terms of a known strain rate $\dot{\mathbf{E}}$. In $2 \mathrm{D}$, this tensor can be expressed as $\dot{\mathbf{E}}=$ $\left[\left(E_{1}, 0\right),\left(0,-E_{1}\right)\right]$. Combining the solutions for the entraining and drainage flow and evaluating equation (9) atop the hemispherical ULVZ patch, the $\mathcal{O}(\epsilon)$ equation becomes

$$
\frac{\mathrm{d} f}{\mathrm{~d} t}=\left(\frac{h^{2}}{2}-1\right) E_{1}+\frac{2}{3 h}\left(\frac{1+\lambda}{2+3 \lambda}\right) \mathcal{R} .
$$

The first term on the right hand side arises from entraining flow, while the 
compare the relative contribution of these two types of flows in determining the shape of the ULVZ.

The critical state at which the drainage and entraining flows balance each other is marked by $\mathrm{d} f / \mathrm{d} t=0$. Notice that in the absence of a straining flow in the ULVZ $\left(E_{1}=0\right)$, the shape change will be determined only by the sign of $\mathcal{R}$. For a dense ULVZ, $\mathcal{R}<0$, rendering $\mathrm{d} f / \mathrm{d} t<0$, such that the top of the ULVZ drains towards the core-mantle boundary. The presence of a straining flow, however, counters this drainage. The critical value $\mathcal{R}_{c}$ for which $\mathrm{d} f / \mathrm{d} t=0$, thus becomes,

$$
\mathcal{R}_{c}=\frac{3 E_{1}}{4}\left(\frac{2+3 \lambda}{1+\lambda}\right) .
$$

The two regimes of flow are defined by the critical compositional Rayleigh number. The plot in Figure 2(b) depicts $\mathcal{R}_{c} / E_{1}$ as a function of $\lambda$ from equation (11). For values of $\mathcal{R} / E_{1}$ above the curve, the shape of the ULVZ is dominated by density contrast-driven drainage flow, while values of $\mathcal{R} / E_{1}$ below the curve lead to the entrainment of the ULVZ by the flow within the LLSVP. In the numerical simulations described in Section 3.2, the flow takes place in the drainage regime.

In the absence of density contrast, the flow takes place entirely in the entrainment regime. We carried out a set of simulations for a neutrally buoyant ULVZ patch located in the corner of the LLSVP. Similar to previously observed behavior of neutrally buoyant, low viscosity blobs embedded in a circulating mantle (Manga, 1996), we found that the neutrally buoyant ULVZs are easily entrained by the mantle circulation and will likely homogenize with the surrounding mantle over geologically significant periods. Results from these simulations are reported in the supplementary material. 


\subsection{Shape of the ULVZs}

While it has been known for some time that the ULVZs are characterized by relatively low thickness (Rost et al., 2006; Rost and Revenaugh, 2003; Williams and Garnero, 1996), recent discoveries of very large $(\sim 800 \mathrm{kms}$ or higher in lateral extent) ULVZs indicate that very high width:height ratio (45:1 to 80:1) can characterize many of the ULVZs (Cottaar and Romanowicz, 2012; Thorne et al., 2013). To understand the development of such flattened shapes as well as the lack of observed ULVZs high above the CMB, we carried out a series of simulations starting with two ULVZ patches suspended above the CMB.

The evolution of the initially suspended ULVZ patches is marked by two stages, leading to the coalescence of the patches. In Figure 3, two initially circular ULVZ patches of $25 \mathrm{~km}$ radius and separated by $100 \mathrm{~km}$, coalesce in two phases. During the initial descent phase spanning over the first $0.75 \mathrm{Ma}$, the dense patches rapidly sink to the bottom of the LLSVP. The vertical drainage within the patches is marked by the mostly vertical streamtubes within each patch. During the next $11 \mathrm{Ma}$ of gravity current phase, the patches spread laterally with a lobate front characteristic of spreading gravity currents (Koch and Koch, 1995; Leahy and Bercovici, 2007). In contrast to the top two panels, the streamtubes within the patches are now almost entirely horizontal. This gravity current phase is also marked by coalescence of the initially distinct patches. As the snapshots in time indicate, the leading patch coats the lobate front of the trailing patch. While such coalescence of dense spreading multiple layers were not observed in previous single gravity current models (Koch and Koch, 1995; Leahy and Bercovici, 2007), the 
coalescence of buoyant viscous blobs rising through a dense liquid follow a similar pattern of coating (Manga and Stone, 1995).

After $12 \mathrm{Ma}$, flow within the LLSVP continues to drag the ULVZ patches towards the corner. At this stage of the simulation, however, highly curved boundary elements lead to the development of numerical instabilities in the simulations. The horizontal stream tubes intersecting the surface of the ULVZ patches indicate that the ULVZs are yet to reach the steady state, as they creep towards the LLSVP corner. To study the steady-state location and shape of the ULVZs, we start the next set of simulations from an initially flat ULVZ patch with rounded corners, described in the following section.

The lack of observed ULVZs far above the CMB and the high width to height ratio is explained by the evolution outlined in Figure 3. While the density contrast rapidly drains any ULVZ patches that might have been created above the CMB, subsequent coalescence of multiple patches explain the recently observed ULVZs of very large lateral extent (Cottaar and Romanowicz, 2012; Thorne et al., 2013). This observation of ULVZ coalescence from our results are qualitatively similar to those obtained by thermochemical convection calculations of McNamara et al. (2010). As described above, the presence of a thin, flat ULVZ patch away from the corner of the LLSVP is likely to be a transient feature of the coupled flow.

\subsection{Stability of the ULVZs}

Upwelling LLSVP circulation sweeps an initially flat ULVZ patch into a steady-state pile near the corner over a geologically short period of time. The second stage of simulation, depicted in Figure 4, starts with a flat ULVZ that would result from the merger of two ULVZ patches to form an approx- 
imately $111 \mathrm{~km}$ long and $9 \mathrm{~km}$ high basal layer. At $8.75 \mathrm{Ma}$, the leading edge of the basal layer is lifted up to a height of $19 \mathrm{~km}$ along the edge of the LLSVP. The change in the topography during the last 3.75 Ma of this simulation is substantially muted compared to the first 1.5 Ma. At 8.75 Ma, the establishment of a closed loop circulation at the left corner of the ULVZ and stream tubes near parallel to the right edge ensures that the shape of the ULVZ has reached near steady-state. The attainment of such a steady-state implies the ULVZ can survive mixing by mantle convection and remain as isolated, chemically distinct entity over geologically significant periods.

To test the stagnation of the ULVZ patch at the corner of the LLSVP, we numerically evaluated the rate of change in topography of the ULVZ patch in the simulation in Figure 4 as a function of time. As the plots in Figure 5 indicate, the initially flat ULVZ patch is uplifted at a velocity of $1.5 \mathrm{~km} / \mathrm{Ma}$ for the first 3.5 Ma. Subsequently the rate of uplift declines rapidly, dropping to zero at $8 \mathrm{Ma}$, indicating the attainment of steady-state topography.

In the third series of reverse experiment, we started with an initial ULVZ patch located at the corner of the LLSVP. The series of simulations in Figure 6 depict the evolution of the shape of the patch. In the beginning of the simulation, the velocity field indicates that the height of the ULVZ is unstable. At the corner, concentric stream tubes depict the closed circulation that was observed in the steady-state in Figure 4 at 8.75 Ma. The circulation on the vertical right edge, however, is quite distinct, as streamtubes enter and leave the ULVZ at the top and the bottom of this edge, respectively. Notice that the stream tubes are tightly bunched over a small fraction of the area of the ULVZ-LLSVP interface, indicating that the flow is focused within narrow 
regions. This counterclockwise flow, driven primarily by buoyancy forces, drains ULVZ material by a gravity current flowing upstream with respect to the entraining flow in the LLSVP. Over the next 2.5 Ma, the gravity current develops a nose-like structure near the bottom, where the outward normal velocity is the highest, indicated by the high angle subtended by the stream tubes to the interface. The opposing flow within the LLSVP lifts the bottom of the right edge of the ULVZ by a small amount, as the gravity current continues spreading upstream. By 5 Ma, two closed circulation cells are established within the ULVZ. Both of these cells are highlighted in the figure. The stream tubes on the ULVZ-LLSVP interface is now mostly tangential to the interface. As a result, the shape of the ULVZ remains practically unchanged during subsequent iterations of the simulation. The steady-state ULVZ has a height of $23.5 \mathrm{~km}$ and width of $67 \mathrm{~km}$ within the corner of the LLSVP. The rate of change in height in Figure 7 demonstrates that a brief initial period of gravitational drainage of the ULVZ is followed by a brief period of uplift, eventually reaching near steady-state around 5Ma.

\subsection{Flow and anisotropy in the ULVZs}

During the coupled flow, the weaker ULVZ absorbs the larger amount of deformation. The plot in Figure 8(a) maps the magnitude of the largest eigenvalue of the strain-rate tensors within the ULVZ and the LLSVP at the steady-state in Figure 4. The warmer colors, corresponding to high strainrate are confined within and around the ULVZ. The strain rate in the interior of the LLSVP is relatively uniform and has a substantially lower value. Low stress levels associated with such low strain rate will confine deformation in diffusion creep regime, preventing the formation of strong anisotropy induced 
by dislocation creep, explaining the observed lack of anisotropy in the LLSVP interior. Strong deformation within and immediately around the ULVZ will lead to the development of a strong textural fabric. If partially molten, such deformation will lead to wetting of grain boundaries by thin films of melt (Daines and Kohlstedt, 1997; Hier-Majumder, 2011; Hier-Majumder and Drombosky, 2015). The map in Figure 8(b) Shows the orientation of strain-rate ellipses within the ULVZ matrix. Partial melt will segregate in films, depicted by the black bars, oriented along the principal axis of shortening (Daines and Kohlstedt, 1997; Hier-Majumder, 2011; Hier-Majumder and Drombosky, 2015) at angles between $30^{\circ}$ and $40^{\circ}$ to the CMB. In addition, high stress levels within the ULVZ can also cause dislocation motion and lattice-preferred orientation in postperovskite crystals.

\section{Assumptions and implications for the lower mantle}

We make two assumptions in our simulations. First, the LLSVP remains stationary over the time period of the simulations. LLSVPs are dynamic structures and may change shape, coalesce, or break-up over geological time scales. Our assumption implies that the timescale for LLSVP migration is larger than the rapid stabilization of the ULVZs. In addition, the boundary condition of tangential velocity at the LLSVP-mantle interface restricts the location of the ULVZ within the physical boundaries of the LLSVPs. Seismic observations of sharp and steep boundaries between the LLSVP and the mantle and geodynamic simulations suggest that the flow within the LLSVP is likely largely self-contained, prompting our selection of the boundary condition at the LLSVP-mantle interface. Isolated observations of ULVZ patches 
away from the LLSVP (McNamara et al., 2010), however, are difficult to explain based on our simulations. To address these issues, future FMBEM models incorporating motion of the LLSVPs need to be constructed. Second, we use a Newtonian linear viscosity for the ULVZ and the LLSVP, any anisotropy arising from the realignment of melt is not taken into account for the simulations. Bearing these assumptions in mind, we discuss the implications of our results for the lower mantle dynamics.

The coupled flow and deformation in the LLSVP-ULVZ system bears a number of implications for the Earth's lowermost mantle. If the ULVZs formed from late stage accumulates of a basal magma ocean (Labrosse et al., 2007; Nomura et al., 2011), they will act as isolated reservoirs of incompatible heat producing elements in the lower mantle (Abe, 1997; Boyet and Carlson, 2005). Such fertile ULVZ reservoirs will also stay close to their solidus temperature at the CMB (Fiquet et al., 2010), containing small amounts of melt, which causes the observed drastic shear wave velocity reduction. As deformation within the ULVZ segregates the melt into thin films subparallel to the CMB, the ULVZs will likely become strongly anisotropic in elastic strength, viscosity, and electrical conductivity. One important outcome of formation of anisotropic melt films will be the development of anisotropic melt permeability. Two recent models of redistribution of melt within the ULVZ demonstrate that shearing from the LLSVPs exert significant influence on compaction and localization of melt (Hernlund and Jellinek, 2010; Hier-Majumder, 2014). Future work on the effect of anisotropic melt distribution within the ULVZ will be crucial in addressing the important issue of melt drainage within the ULVZ. Finally, here we explore the commonly 
observed association between LLSVP corners and ULVZ patches. But thin, undetectable ULVZ layers can exist outside the LLSVPs. ULVZs observed outside the LLSVP are likely associated with regions of localized mantle upwelling (Jellinek and Manga, 2004).

In conclusion, our results demonstrate that dense, low-viscosity ULVZs stagnate at the corner of LLSVPs remaining chemically unmixed over geologically long periods of time. Away from the LLSVP corners, initially distinct ULVZ patches can coalesce within a few Ma into flat structures near immediately above the CMB. During the coupled flow of the surrounding mantle and the ULVZ, most of the deformation is partitioned within and around the ULVZ, explaining the lack of observed anisotropy in the interior of the LLSVP.

\section{Acknowledgments}

This work was supported by NSF grant EAR 1215800 and XSEDE computational grant EAR130030. Mark Jellinek, John Brodholt, and anonymous reviewer provided insightful reviews. 


\section{Appendix A. Linear stability analysis}

In this section, we present the method for building an analytical solution to the velocity and pressure fields within the ULVZ and the evolution of ULVZ shape subjected to a flow within the surrounding mantle.

Treating the ULVZ and the mantle as separate entities, we notice that mass and momentum is conserved within each of them. The conservation of mass is described by equation (1). Since we treat pressure as a primary variable in this analysis, we can recast the equation for conservation of momentum as,

$$
-\nabla p^{j}+\mu^{j} \nabla^{2} \boldsymbol{u}^{j}=0,
$$

where superscript $j=U$ for the ULVZ and $L$ for the LLSVP. We can write the perturbed velocity field within each entity as $\boldsymbol{u}^{j}=\epsilon\left(\boldsymbol{u}_{e}^{j}+\boldsymbol{u}_{d}^{j}\right)$, where $\epsilon \ll 1$ and the subscripts $e$ and $d$ refer to two linear flows characteristic of entrainment and drainage. We build analytical solutions for both of these velocity fields separately. Since the overall nature of the flow is linear, superposition of two different flow fields is justified. This formulation enables us to determine the relative importance between drainage and entrainment.

For the draining flow, we use the Hadamard-Rybczinski solution, for a translating flow past a viscous sphere (Leal, 1992, Ch. 5). For the entraining flow, we build analytical solutions for velocity and pressure fields using solid vector harmonics. The system of solution building using the method of solid harmonics is described in a number of previous articles (Drombosky and Hier-Majumder, 2015; Hier-Majumder, 2011; Hier-Majumder and Drombosky, 2015).

To study the influence of deformation of the ULVZ, it is useful to build 
the solutions in terms of a dimensionless, constant strain rate tensor $\dot{\mathbf{E}}$. We set the origin at the center of the hemispherical ULVZ, such that the position vector $\boldsymbol{r}$ describes the distance of a point from the center of the ULVZ. The nondimensional velocity and pressures within the ULVZ and the LLSVP are then given by

$$
\begin{aligned}
\boldsymbol{u}_{s}^{U} & =-a_{1}\left(1-r^{2}\right) \dot{\mathbf{E}} \cdot \boldsymbol{r}+a_{2} \boldsymbol{r} \dot{\mathbf{E}}: \boldsymbol{r} \boldsymbol{r} \\
p_{s}^{U} & =a_{3} \frac{\lambda}{2} \dot{\mathbf{E}}: \boldsymbol{r} \boldsymbol{r} \\
\boldsymbol{u}_{s}^{L} & =c_{1} \dot{\mathbf{E}} \cdot \boldsymbol{r} \\
p_{s}^{L} & =c_{2} \dot{\mathbf{E}}: \boldsymbol{r} \boldsymbol{r} .
\end{aligned}
$$

In the Cartesian coordinates, we can describe the strain tensor $\dot{\mathbf{E}}$ in terms the principal strain rates as,

$$
\dot{\mathbf{E}}=\left[\begin{array}{ll}
E_{1} & 0 \\
0 & -E_{1}
\end{array}\right] \text {. }
$$

The solution for $\boldsymbol{u}_{s}^{L}$ ensures the conservation of mass within the LLSVP. We also rewrite the stress jump condition as,

$$
\lambda \hat{\boldsymbol{n}} \cdot \mathbf{T}_{s}^{U} \cdot \hat{\boldsymbol{n}}-\hat{\boldsymbol{n}} \cdot \mathbf{T}_{s}^{L} \cdot \hat{\boldsymbol{n}}=0,
$$

at $r=h$.

We determine four of the five unknown constants from mass and momentum conservation within the ULVZ (equations (1) and (A.1)), momentum conservation within the LLSVP (equation (A.1)) and continuity of normal traction across the ULVZ-mantle interface (equation (A.7)). Then, setting 
$c_{1}=\lambda$, we obtain the following constants,

$$
\begin{aligned}
& a_{1}=-1 \\
& a_{2}=\frac{1}{2} \\
& a_{3}=-6 \\
& c_{1}=\lambda \\
& c_{2}=0 .
\end{aligned}
$$

456 Solution for the axisymmetric Hadamard-Rybczinski flow is given is spher${ }_{457}$ ical polar coordinates (Kim and Karilla, 2005, Ch. 4). For brevity, we only provide the solutions for flow internal to the ULVZ, which will be used in the next step. This solution is given by,

$$
\begin{aligned}
\boldsymbol{u}_{\boldsymbol{d}}^{\boldsymbol{L}}= & {\left[-\frac{1}{2(1+\lambda)}\left(\frac{r}{h}\right)^{2}+\frac{3+2 \lambda}{2(1+\lambda)}\right] U \cos \theta \hat{\boldsymbol{r}} } \\
& -\left[\frac{1}{1+\lambda}\left(\frac{r}{h}\right)^{2}+\frac{3+2 \lambda}{2(1+\lambda)}\right] U \sin \theta \hat{\boldsymbol{\theta}}
\end{aligned}
$$

460 where $\hat{\boldsymbol{r}}$ and $\hat{\boldsymbol{\theta}}$ are unit vectors and $U$ is a constant terminal velocity, respec461 tively. Notice that atop the $\operatorname{ULVZ}(r=h, \theta=0)$ this velocity reduces to $462 U \hat{\boldsymbol{r}}$. The dimensional magnitude of the terminal velocity, $U^{\prime}$, can be modified 463 from (Leal, 1992, Eq. 5.45) using our definitions

$$
U^{\prime}=\frac{2}{3}\left(\frac{1+\lambda}{2+3 \lambda}\right) \frac{x_{c}^{2} g \Delta \rho}{\mu} .
$$

464 Using the characteristic velocity for nondimensionalization, we can rewrite 465 $U$ in terms of the dimensionless compositional Rayleigh number as,

$$
U=\frac{2}{3}\left(\frac{1+\lambda}{2+3 \lambda}\right) \mathcal{R} .
$$


The solution for straining flow leads to the following expression for the normal component of the velocity atop the $\operatorname{ULVZ}(r=h, \theta=0)$

$$
\boldsymbol{u}_{s}^{U} \cdot \hat{\boldsymbol{r}}=\left(\frac{h^{3}}{2}-h\right) E_{1} .
$$

To determine the change of the ULVZ shape atop, we rewrite equation (8) in $\mathcal{O}(\epsilon)$ as,

$$
\frac{\partial f(\boldsymbol{x}, t)}{\partial t}=\frac{1}{h} \boldsymbol{u}^{U}(\boldsymbol{x}) \cdot \hat{\boldsymbol{n}} .
$$

Decomposing the total velocity $\boldsymbol{u}^{U}$ in terms of the entraining velocity $\boldsymbol{u}_{s}^{U}$ and the draining velocity $\boldsymbol{u}_{d}^{U}$, and using the expressions from equations (A.13) and (A.16) for $r=h, \theta=0$, we can rewrite equation (A.17) as,

$$
\frac{\mathrm{d} f}{\mathrm{~d} t}=\left(\frac{h^{3}}{2}-h\right) E_{1}+\frac{2}{3}\left(\frac{1+\lambda}{2+3 \lambda}\right) \mathcal{R} .
$$

In the main article, we discuss the nature of the above equation in determining the regime boundary between entrainment and drainage.

\section{References}

Abe, Y., 1997. Thermal and chemical evolution of the terrestrial magma ocean. Physics of Earth and Planetary Interiors 100, 27-39.

Bower, D. J., Wicks, J. K., Gurnis, M., Jackson, J. M., 2011. A geodynamic and mineral physics model of a solid-state ultralow-velocity zone. Earth and Planetary Science Letters 303 (3-4), 193 - 202.

URL http://www.sciencedirect.com/science/article/pii/S0012821X10008083

Boyet, M., Carlson, R. W., 2005. 142 Nd Evidence for Early (¿4.53 Ga)

Global Differentiation of the Silicate Earth. Science (July), 576-581. 
Coltice, N., Moreira, M., Hernlund, J., Labrosse, S., 2011. Crystallization of a basal magma ocean recorded by helium and neon. Earth and Planetary Science Letters 308 (1-2), 193 - 199.

URL http://www.sciencedirect.com/science/article/pii/S0012821X11003384

Cottaar, S., Romanowicz, B., Nov. 2012. An unsually large ULVZ at the base of the mantle near Hawaii. Earth and Planetary Science Letters $355-356,213-222$.

URL http://linkinghub.elsevier.com/retrieve/pii/S0012821X12005006

Cottaar, S., Romanowicz, B., Aug. 2013. Observations of changing anisotropy across the southern margin of the African LLSVP. Geophysical Journal International 195 (2), 1184-1195.

URL http://gji.oxfordjournals.org/cgi/doi/10.1093/gji/ggt285

Daines, M. J., Kohlstedt, D. L., 1997. Influence of deformation in melt topology in peridotites. Journal of Geophysical Research 102 (B5), $10257-$ 10271.

Drombosky, T. W., Hier-Majumder, S., 2015. Development of anisotropic texture in deforming partially molten aggregates i: Theory and fast multipole boundary elements method. Journal of Geophysical Research, Solid Earth (in press) 120.

Fiquet, G., Auzende, A. L., Siebert, J., Corgne, A., Bureau, H., Ozawa, H., Garbarino, G., 2010. Melting of peridotite to 140 Gigapascals. Science 329 (5998), 1516-8. 
Hernlund, J. W., Jellinek, A. M., JUL 15 2010. Dynamics and structure of a stirred partially molten ultralow-velocity zone. Earth and Planetary Science Letters 296 (1 - 2), $1-8$.

Hier-Majumder, S., DEC 11 2008. Influence of contiguity on seismic velocities of partially molten aggregates. Journal of Geophysical Research-Solid Earth 113 (B12), B12205.

Hier-Majumder, S., 2011. Development of anisotropic mobility during twophase flow. Geophysical Journal International 186, 59-68.

Hier-Majumder, S., 2014. Melt redistribution by pulsed compaction within ultralow velocity zones. Phys. Earth and Planet. Int. 229, 134-143.

Hier-Majumder, S., Abbott, M. E., OCT 15 2010. Influence of dihedral angle on the seismic velocities in partially molten rocks. Earth and Planetary Science Letters 299 (1-2), 23-32.

Hier-Majumder, S., Drombosky, T. W., 2015. Development of anisotropic texture in deforming partially molten aggregates ii: Implications for the lithosphere-asthenosphere boundary. Journ. Geophys. Res. 120.

Hier-Majumder, S., Revenaugh, J., NOV 1 2010. Relationship between the viscosity and topography of the ultralow-velocity zone near the core-mantle boundary. Earth and Planetary Science Letters 299 (3-4), 382-386.

Jellinek, A., Manga, M., 2004. Links between long-lived hotspots, mantle plumes D", and plate tectonics. Reviews of Geophysics 42 (RG3002). 
Kim, S., Karilla, S. J., 2005. Microhydrodynamics: Principles and selected applications. Dover, Ch. 13.

Koch, D. M., Koch, D. L., Apr. 1995. Numerical and theoretical solutions for a drop spreading below a free fluid surface. Journal of Fluid Mechanics $287,251-278$.

URL http://www . journals. cambridge.org/abstract_S0022112095000942

Labrosse, S., Hernlund, J., Coltice, N., 2007. A crystallizing dense magma ocean at the base of earth's mantle. Nature 450, 866-869.

Leahy, G., Bercovici, D., 2007. On the dynamics of a hydrous melt layer above the transition zone. Journal of Geophysical Research 112 (B07401).

Leal, G., 1992. Laminar Flow and Convective Transport Processes. Butterworth-Heinemann.

Liu, Y., Nishimura, N., MAY 2006. The fast multipole boundary element method for potential problems: A tutorial. ENGINEERING ANALYSIS WITH BOUNDARY ELEMENTS 30 (5), 371-381.

Lynner, C., Long, M., 2014. Lowermost mantle anisotropy and deformation along the boundary of the african llsvp. Geophys. Res. Lett.

Manga, M., 1996. Mixing of heterogeneities in the mantle: Effect of viscosity differences. Geophysical Research Letters 23 (4), 403-406.

Manga, M., Stone, H., 1995. Low reynolds number motion of bubbles, drops, and rigid spheres through fluid-fluid interfaces. Journal of Fluid Mechanics 287, 279-298. 
Mao, W. L., Mao, H.-K., Sturhahn, W., Zhao, J., Prakapenka, V. B., Meng, Y., Shu, J., Fei, Y., Hemley, R. J., 2006. Iron-rich post perovskite and the origin of the ultra low velocity zone. Science $312,564-565$.

McNamara, A. K., Garnero, E. J., Rost, S., OCT 15 2010. Tracking deep mantle reservoirs with ultra-low velocity zones. Earth and Planetary Science Letters 299 (1-2), 1-9.

Muir, J. M., Brodholt, J. P., 2015a. Elastic properties of ferropericlase at lower mantle conditions and its relevance to ULVZs. Earth and Planetary Science Letters 417, 40-48.

URL http://linkinghub.elsevier.com/retrieve/pii/S0012821X15001090

Muir, J. M. R., Brodholt, J. P., 2015b. Elastic properties of ferrous bearing MgSiO3 and their relevance to ULVZs. Geophysical Journal International 201 (1), 496-504.

URL http://gji.oxfordjournals.org/cgi/doi/10.1093/gji/ggv045

Nomura, R., Ozawa, H., Tateno, S., Hirose, K., Hernlund, J., Muto, S., Ishii, H., Hiraoka, N., MAY 12 2011. Spin crossover and iron-rich silicate melt in the Earth's deep mantle. NATURE 473 (7346), 199+.

Olson, P., 1990. Hot spots, swells, and mantle plumes. In: Ryan, M. P. (Ed.), Magma Transport and Storage. AGU, pp. 33-51.

Pozrikidis, C., 2001. Interfacial dynamics for stokes flow. Journal of Computational Physics 169, 250-301.

Rost, S., Garnero, E. J., Williams, Q., 2006. Fine-scale ultralow-velocity zone 
structure from high frequency seismic array data. Journal of Geophysical Research, B 111 (B09310).

Rost, S., Garnero, E. J., Williams, Q., Manga, M., 2005. Seismological constraints on a possible plume root at the core-mantle boundary. Nature 435, 666-669.

Rost, S., Revenaugh, J., 2003. Small scale ultralow-velocity zone structure imaged by ScP. Journal of Geophysical Research 108 (B12056).

Saad, Y., 2003. Iterative Methods for Sparse Linear Systems, 2nd Edition. SIAM.

Thorne, M. S., Garnero, E. J., Jahnke, G., Igel, H., McNamara, A. K., Feb. 2013. Mega ultra low velocity zone and mantle flow. Earth and Planetary Science Letters 364, 59-67.

URL http://linkinghub.elsevier.com/retrieve/pii/S0012821X12007200

Wicks, J. K., Jackson, J. M., Sturhahn, W., Aug. 2010. Very low sound velocities in iron-rich ( $\mathrm{Mg}, \mathrm{Fe}) \mathrm{O}$ : Implications for the core-mantle boundary region. Geophysical Research Letters 37 (15), 1-5.

URL http://www .agu.org/pubs/crossref/2010/2010GL043689.shtml

Williams, Q., Garnero, E., 1996. Seismic evidence for partial melt at the base of Earth's mantle. Science 273, 1528-1530.

Wimert, J. T., Hier-Majumder, S., 2012. A three-dimensional microgeodynamic model of melt geometry in the earth's deep interior. Journal of Geophysical Research-Solid Earth 117 (B04), B04203. 


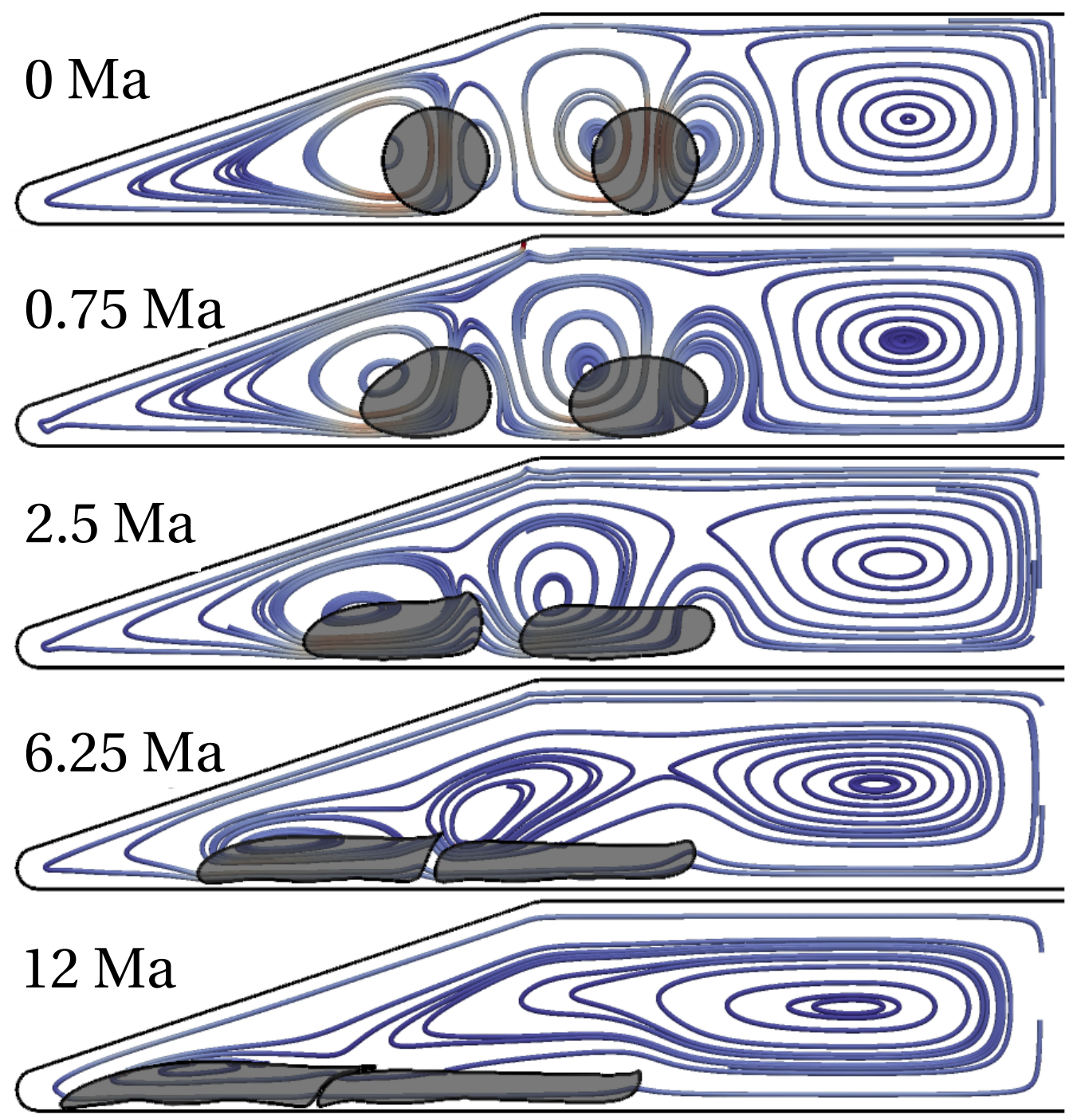

Figure 3: Evolution of circular ULVZ reservoirs placed in the LLSVP. The five snapshots show the evolution of the system between 0 and $12 \mathrm{Ma}$. The streamlines are colored by the magnitude of the velocity with light blue corresponding to $1 \mathrm{~cm} / \mathrm{yr}$ and dark red to 3 $\mathrm{cm} / \mathrm{yr}$. The $10 \%$ denser and two orders of magnitude weaker ULVZ patches are shaded in gray. Only half of the symmetric LLSVP flow is shown in the figure. 


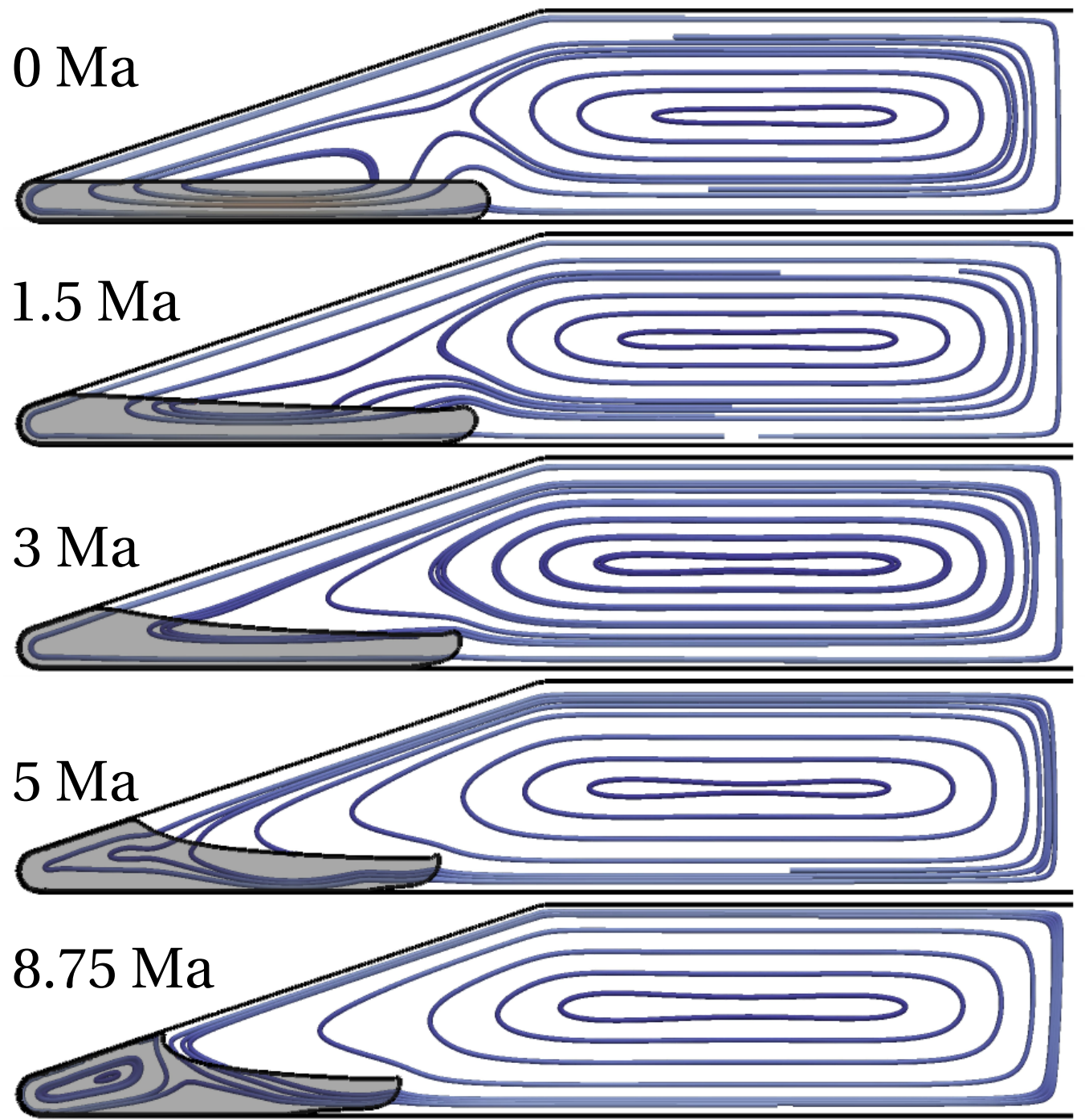

Figure 4: Evolution of rectangular ULVZ reservoirs with rounded corners placed in corners of the LLSVP. The five snapshots show the evolution of the system between 0 and $8.75 \mathrm{Ma}$. The streamlines are colored by the magnitude of the velocity with light blue corresponding to $1 \mathrm{~cm} / \mathrm{yr}$ and dark red to $2 \mathrm{~cm} / \mathrm{yr}$. The ULVZ patches are separated from the LLSVP by a black outline and shaded in gray. At $8 \mathrm{Ma}$, the rate of change of ULVZ topography becomes zero, indicating steady-state. 


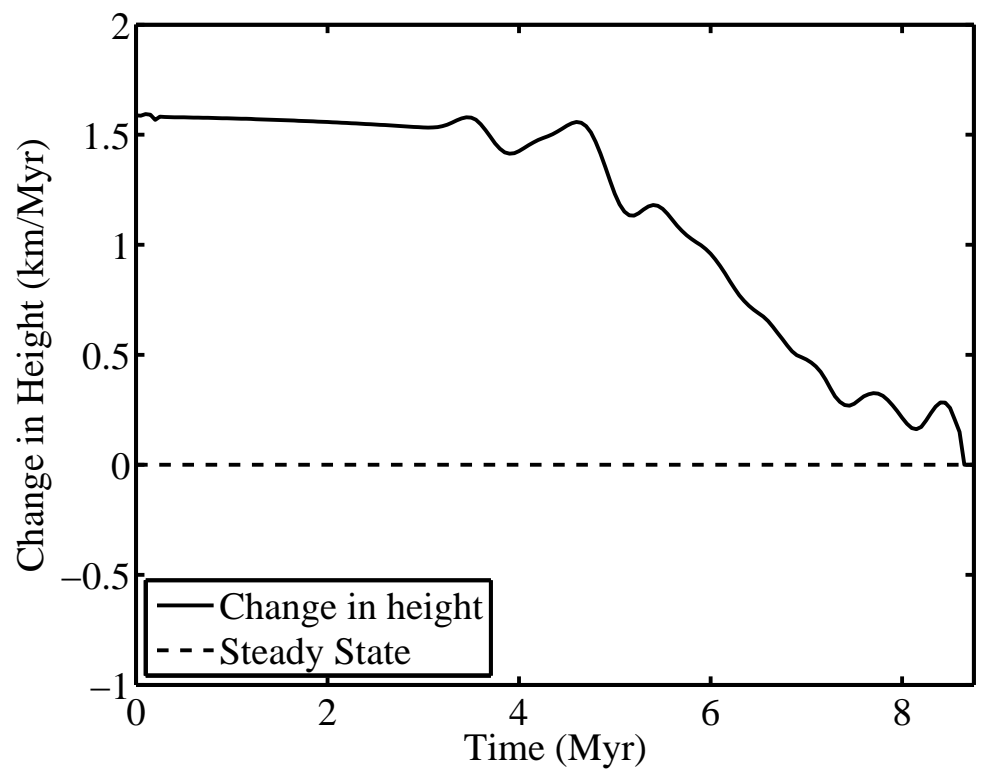

Figure 5: Rate of change of the ULVZ height in $\mathrm{km} / \mathrm{Ma}$ is plotted from the numerical simulation in Figure 4 in solid line. The dashed line represents no change in height, i.e. steady state. The derivative is passed through a triangle smoothing filter to reduce noise in the measurement. 

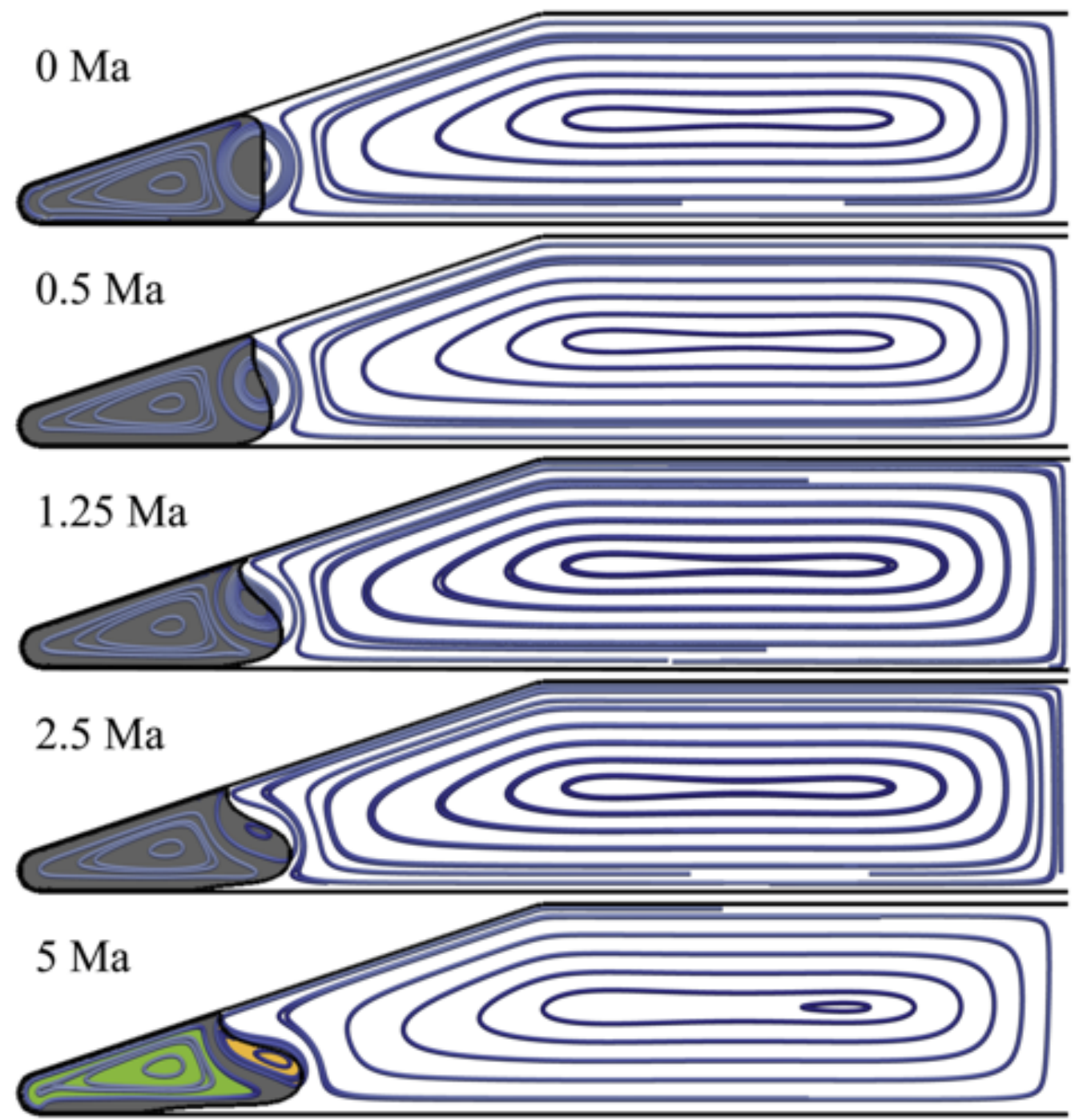

Figure 6: Evolution of ULVZ reservoirs wedged into the corners of the LLSVP. The five snapshots show the evolution of the system between 0 and 5 Ma. The streamlines are colored by the magnitude of the velocity with light blue corresponding to $1 \mathrm{~cm} / \mathrm{yr}$ and dark red to $2 \mathrm{~cm} / \mathrm{yr}$. The ULVZ patches are separated from the LLSVP by the black outline and shaded in gray. The last snapshot highlights the streamlines, in green and orange, completely within the ULVZ indicating a steady state geometry has been achieved. 


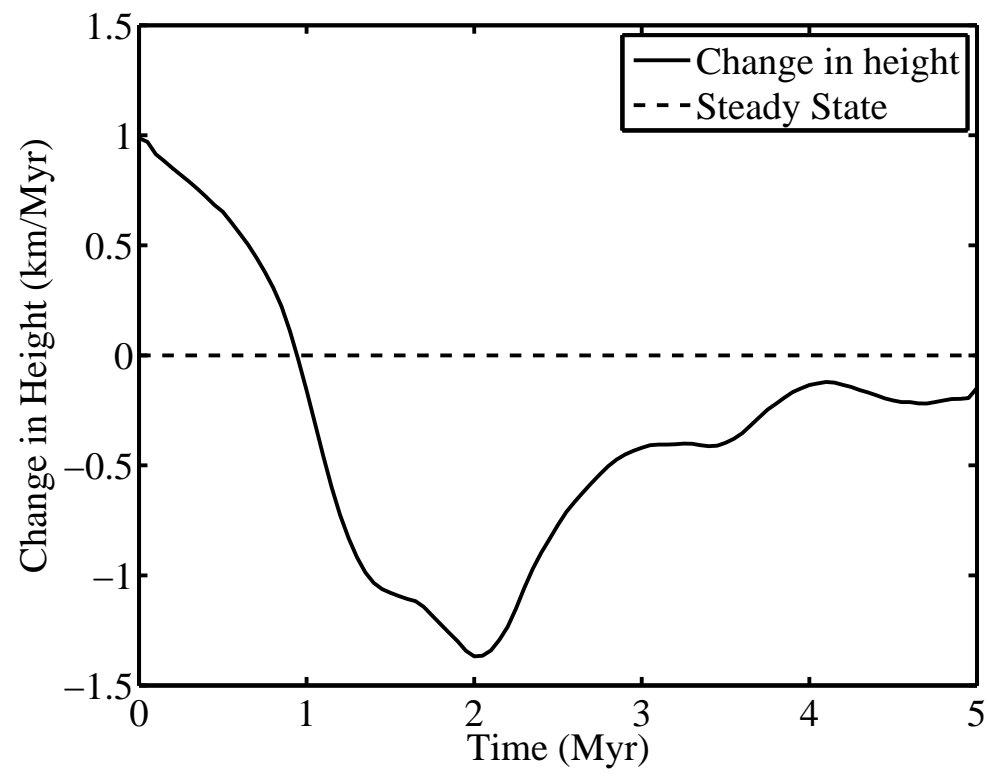

Figure 7: Change in height of the ULVZ reservoir as it falls down the wall of the LLSVP in Figure 6 is plotted in the solid line. The dashed line represents no change in height, i.e., steady state. The derivative is passed through a triangle smoothing filter to reduce noise in the measurement. 


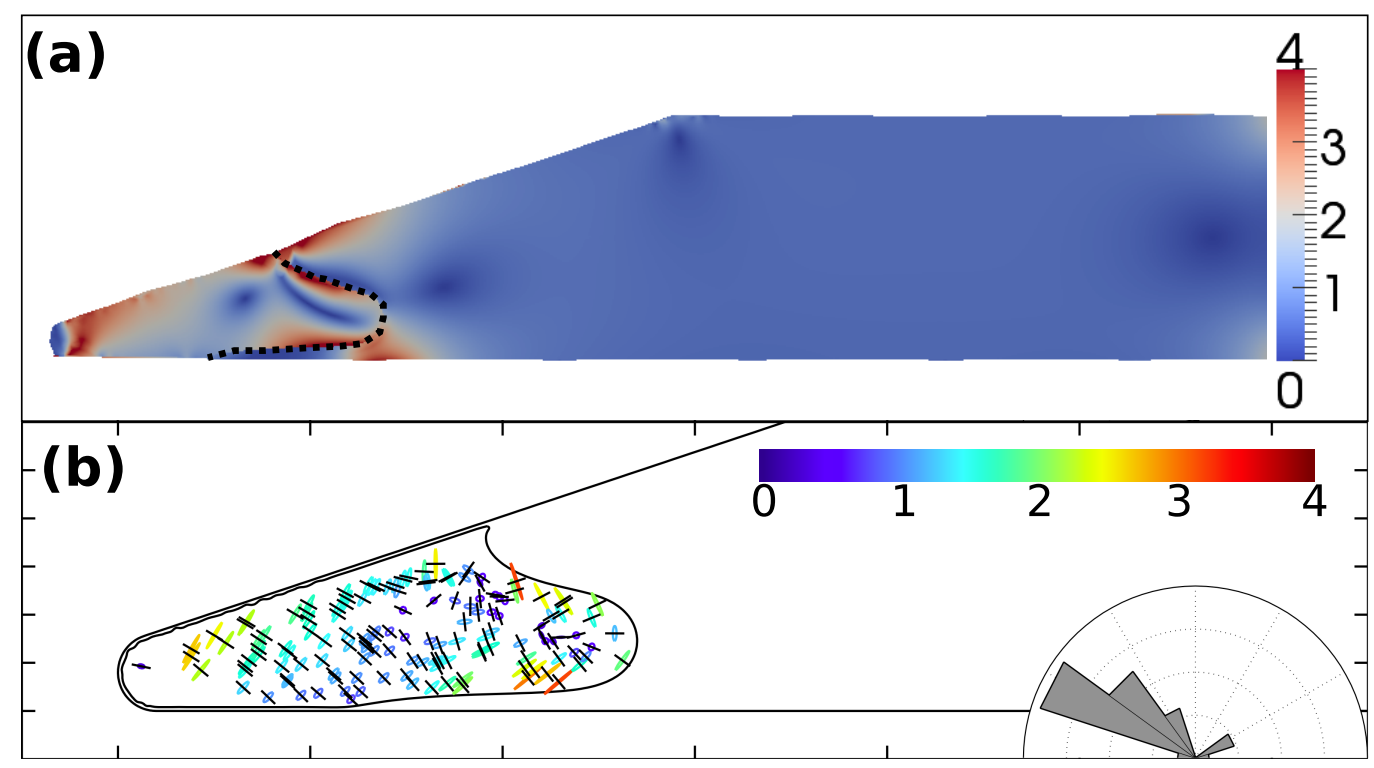

Figure 8: (a) Map of the magnitude of the largest principal strain rate within the coupled ULVZ-LLSVP system. The dotted line outlines the steady-state ULVZ-LLSVP interface at the end of the series of simulations in Figure 2 of the supplementary material. (b) Orientation of principal strain ellipses, colored by the magnitude of the largest principal strain rate. The black bars are oriented parallel to the principal shortening axis, and serve as a proxy for the orientation of melt films. The rose diagram in the inset depicts the frequency of angles subtended by the melt films with the CMB. 